\title{
Detection and Classification of Mu Rhythm using Phase Synchronization for a Brain Computer Interface
}

\author{
Oana Diana Eva \\ Faculty of Electronics, Telecommunications and Information Technology \\ "Gheorghe Asachi" Technical University \\ Institute of Computer Science \\ Romanian Academy \\ Iasi, Romania
}

\begin{abstract}
Phase synchronization in a brain computer interface based on Mu rhythm is evaluated by means of phase lag index and weighted phase lag index. In order to detect and classify the important features reflected in brain signals during execution of mental tasks (imagination of left and right hand movement), the proposed methods are implemented on two datasets. The classification is performed using linear discriminant classifier, quadratic discriminant classifier, Mahalanobis distance classifier, $k$ nearest neighbor and support vector machine. Classification accuracies up to $74 \%$ and $61 \%$ for phase lag index and weighted phase lag index were achieved. The results indicate that phase synchronization measures are relevant for classifying mental tasks recorded in the active state and the relaxation state from additional motor area and from the sensorimotor area. Phase lag index and weighted phase lag index methods are easy to implement, efficient, provide relevant features for the classification and can be used as an offline methods for motor imagery paradigms.
\end{abstract}

Keywords-brain computer interface; electroencephalogram; phase synchronization; phase lag index; weighted phase lag index; classifiers

\section{INTRODUCTION}

Brain computer interface (BCI) systems translate brain activities into commands for external devices. Their main goal to provide a communication channel for people with severe motor disabilities.

One of the most popular and used method in recording neurological signals is the electroencephalogram (EEG). It is simple to use, implies low costs and has a very high time resolution allowing EEG based BCIs to respond very quickly to user commands [1].

EEG based BCIs identify changes that occur while the person performs different mental tasks and make use of important features in classification. The linear classifiers, neural networks and nearest neighbor classifiers are most used in BCI applications.

The EEG signals contain amplitude and phase information. Common spatial pattern (CSP) [2], power spectral density (PSD) [1], adaptive auto regressive (AAR) parameters [3] and Hurst exponent [4] have been used to extract from EEG amplitude distinctive features in different mental states. Phase synchrony is a mechanism for dynamic integration of distributed neural networks in the brain. Phase relationships identified between the recordings of electrophysiological activity generated within different cortical regions may provide information about functional connections between those cortical regions [5]. Such relationships are reported between cortical regions that are used in the control of a BCI where spectral coherence quantifies phase synchronization for electrophysiological data [6].

Phase synchronization can be difficult due to the presence of the common reference, the volume-conduction of source activity and the presence of noise sources [7]. To overcome these problems, methods that are focusing on phase instead of the amplitude have been proposed: the phase locking value (PLV) [8], that uses only the relative phase between signals to measure the phase-synchronization, the imaginary component of the coherency (ImC) [9] as a conservative index of phasesynchronization, phase lag index (PLI) [10] as a potential improvement of the ImC and the weighted phase lag index (WPLI) [4] in order to increase the capacity to detect true changes in phase synchronization.

Sensorimotor rhythms (SMR) refer to a brain oscillation in the specific frequency band generated from the sensorimotor area. SMRs are modulated by motor execution, motor observation and motor imagery [11].

While performing a mental activity as left/right hand movement or imagination changes appear in the sensorimotor area in the corresponding signal power of $\mathrm{Mu}(8-12 \mathrm{~Hz})$ and Beta $(12-30 \mathrm{~Hz})$ rhythms.

An off-line analysis is performed in order to extract and classify significant features contained in the EEG signals using indexes that characterize the phase synchronization (PLI and WPLI) in a BCI paradigm based on sensorimotor rhythms. Five classifiers are used for distinguishing mental tasks.

In the section II databases and methods used are described, how the features are extracted and how the classification methods are applied. The results are depicted in section III. The paper ends with discussions based on our results (section IV) and conclusions (section V). 


\section{MethodOLOGY}

\section{A. Datasets}

The EEG signals are recorded with a portable acquisition system from the lab of the Faculty of Medical Bioengineering. It is provided by g.tec medical engineering $\mathrm{GmbH}$ and is based on a g.GAMMAcap unit, a g.GAMMAbox unit and a g.MOBIlab+ one. The sampling frequency of $256 \mathrm{~Hz}$ and BCI2000 platform [12] are used. 8 electrodes (CP3, CP4, P3, P4, C3, C4, PZ and CZ) are placed on the subject scalp according to International 10-20 System. The reference electrode is linked on the right earlobe.

Subjects (33 healthy volunteers, men and women, age range 19-59 years) are asked to perform motor imagery tasks. The subjects are sitting relaxed in front of a computer screen. At the beginning of a trial, the screen is white. After $2 \mathrm{~s}$, an arrow pointing to the left or to the right appears on the screen. The subject has to imagine the hand movement indicated by the arrow and to relax when the screen is white. During the recordings, subjects are advised to try to avoid the eye blinking, eye movements, feet movements or swallowing. Each arrow appears up for 30 times. The trials are conducted in different days. All subjects provide written informed consent prior to the experiment.

The second dataset used is provided by Dr. Allen Osman in BCI Competition 2002 [13]. The EEGs are recorded by 59 electrodes. The signals are sampled at the frequency $100 \mathrm{~Hz}$. The reference electrode is placed on the left mastoid. The dataset consists of EEG data recorded from 9 well trained subjects. Each session lasts $6 \mathrm{~s}$ and each run consists of 90 trials (45 trials for imagery of left hand movement and 45 for imagery of right hand movement). In each trial a cue is shown on the screen instructing the subject to perform one of the following motor imagery tasks: left hand, right hand, relaxation.The signals acquired from 9 electrodes (FC3, FCZ, FC4, CP3, CPZ, CP4, C3, CZ and C4) over the sensorimotor area are considered for further processing.

\section{B. Methods}

The phase lag index and the weighted phase lag index are used for measuring the synchronization between two signals $x(t)$ and $y(t)$.

The phase lag index measures the statistical interdependencies between time series. The aim of PLI is to obtain reliable estimates of phase synchronization that are invariant against the presence of common sources as active reference electrodes.

The asymmetry of the phase difference distribution means that the likelihood that the phase difference $\Delta \theta$ will be in the interval $-\pi<\Delta \theta<0$ is different from the likelihood that it will be in the interval0 $<\Delta \theta<\pi$. This asymmetry implies the presence of a nonzero phase difference ('lag') between the two time series [10].

An index of the asymmetry of the phase difference distribution can be obtained from a time series of phase differences $\Delta \theta\left(t_{k}\right), k=1 \ldots N$ in the following way:

$$
P L I=\left|\left\langle\operatorname{sign}\left[\Delta \theta\left(t_{k}\right)\right]\right\rangle\right|,
$$

where sign is the signum function and $-\pi \leq \Delta \theta \leq+\pi$.

The PLI ranges between 0 (no interaction) and 1 (maximum interaction), $0 \leq P L I \leq 1$. The $<>$ denotes the average over the time $t$. A PLI of zero indicates either no coupling or coupling with a phase difference $\Delta \theta$ centered around $[0, \pi]$ [10].

The WPLI increases the capacity to detect real changes that occur in phase synchronization, reduces the influence of common noise sources and reduces the influence of changes in the coherence phase [7]. The weighted phase lag index is calculated using the formula:

$$
W P L I=|\langle I(X)\rangle| /\langle|I(X)|\rangle=|\langle I|X| \operatorname{sign} I(X)\rangle| /\langle|I(X)|\rangle
$$

where $I(X)$ is the imaginary component of the cross spectrum between two signals $x(t)$ and $y(t)$.

The complex cross-spectrum $C(f)$ for two real-valued signals $x(t)$ and $y(t)$ is computed by Fourier transform.

$$
C(f)=\left\langle X(f) Y^{*}(f)\right\rangle,
$$

where $X(f)$ is the Fourier transform of signal $x(t)$ and $Y(f)$ is the Fourier transform of signal $y(t)$. The symbol $*$ indicates the complex conjugation and \langle\rangle denotes the expectation value.

The values of the WPLI are ranged between 0 and 1, where 1 means total synchronization. Synchronization is defined by $P\{\operatorname{sign}(I(X))=1\}=1$ or $P\{\operatorname{sign}(I(X))=-1\}=1$, where $P\{$.$\} denotes probability. The WPLI is based on the imaginary$ component of the cross spectrum [7].

\section{Preprocessing of the EEG signals}

All EEG signals from the first dataset are loaded, and then they are band-pass filtered in the $8-12 \mathrm{~Hz}$ frequency band by means of 100th order Finite Impulse Response (FIR) filter in order to avoid phase distortions. Data segments corresponding to the left hand motor imagery and to the right hand motor imagery are extracted.

New obtained EEG signals are analyzed using the PLI and the WPLI. To compute the phase synchronization, it is necessary to know the instantaneous phase of the two signals involved. This can be realized using the Hilbert transform. The Hilbert transform is performed for all the channels (CP3, CP4, P3, P4, C3, C4, PZ and CZ) and the PLI and the WPLI are computed for all the possible pairs of EEG channels (both for left hand motor imagery and for right hand motor imagery).

Two electrodes from the additional motor area $\mathrm{CZ}$ and $\mathrm{PZ}$ and six electrodes from the sensorimotor areas P3, C3, CP3 (from left hemisphere) and $\mathrm{P} 4, \mathrm{C} 4, \mathrm{CP} 4$ (from right hemisphere) are used. Six combinations for CZ (CZ-CP3, CZCP4, CZ-P3, CZ-P4, CZ-C3, CZ-C4), six combinations for PZ (PZ-CP3, PZ-CP4, PZ-P3, PZ-P4, PZ-C3, PZ-C4) and three combinations left-right (CP3-CP4, P3-P4 and $\mathrm{C} 3-\mathrm{C} 4)$ are extracted. WPLI.

The approach is firstly evaluated using the PLI and then the

The same steps are followed for the second dataset but other channels are handled. Nine combinations for left 
hemisphere: FCZ-FC3, FCZ-C3, FCZ-CP3, CZ-FC3, CZ-C3, CZ-CP3, CPZ-FC3, CPZ-C3, CPZ-CP3, nine combinations for right hemisphere: FCZ-FC4, FCZ-C4, FCZ-CP4, CZ-FC4, CZC4, CZ-CP4, CPZ-FC4, CPZ-C4, CPZ-CP4 and three combinations left-right: $\mathrm{CP} 3-\mathrm{CP} 4, \mathrm{FC} 3-\mathrm{FC} 4$ and $\mathrm{C} 3-\mathrm{C} 4$ are formed.

\section{Classifiers}

In order to observe the discrimination between left or right motor activity we used 5 classifiers: linear discriminant analysis (LDA) [6], quadratic discriminant analysis (QDA) [14], Mahalanobis distance (MD) [15], k nearest neighbor (KNN) [16] and support vector machine (SVM) [17]. A 10x10 fold cross validation estimated the classification rate for each subject.

The feature vector is formed by PLI indexes for left motor imagery and right motor imagery. Data are divided into 10 parts with an equal number of features, 5 parts are used for training and the other parts for testing.

\section{RESULTS}

Fig. 1 displays the classification rates obtained with classifiers LDA, QDA and MD for PLI for the dataset developed with EEGs recorded in our lab. The classification rates are above 60\%. 68\% from the subjects obtained better results with QDA classifier and the others 32\% with MD classifier.

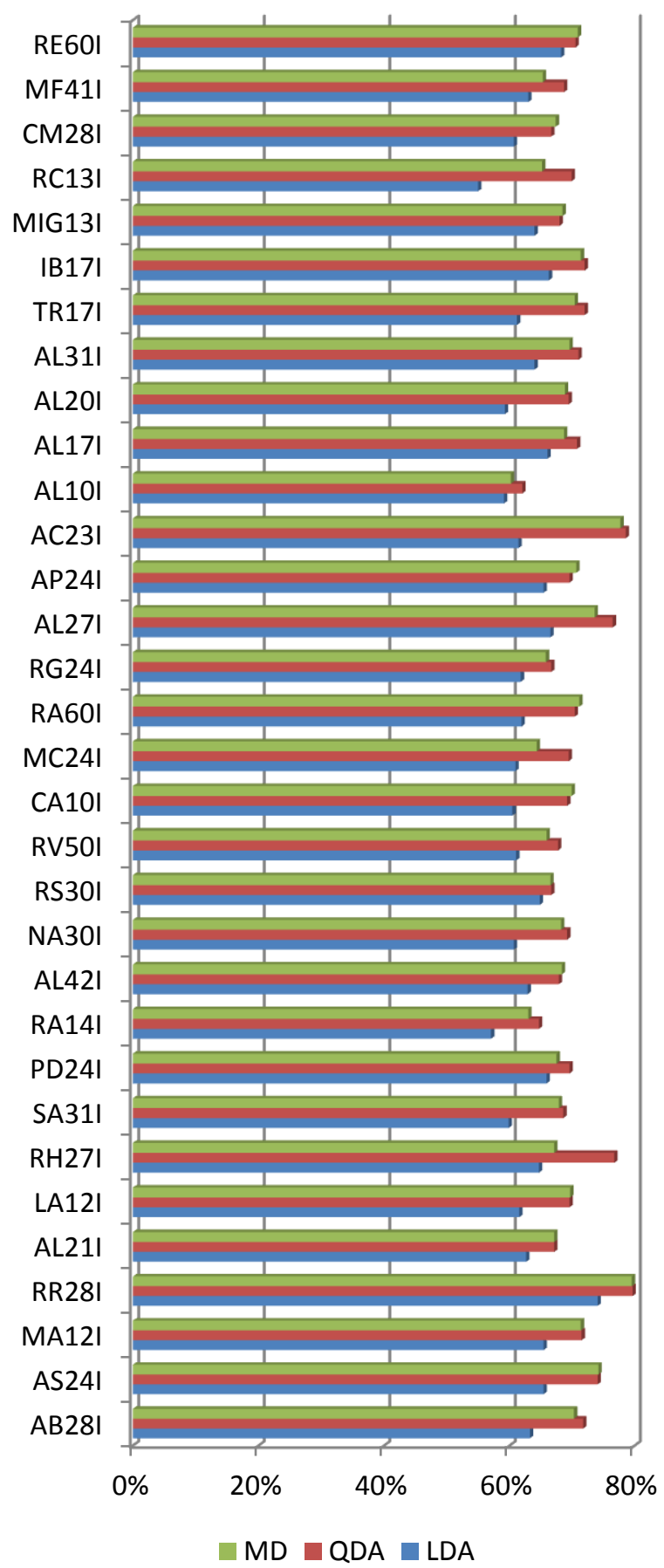

Fig. 1. The classification rates of PLI using classifiers LDA, QDA and MD 
TABLE I. shows the classification rates using KNN classifier for 1, 2, 3, 4 or 5 neighbors. The higher classification rates of about $83 \%$ are obtained by subject $\mathrm{RH} 27 \mathrm{I}$ and the smaller rates of about $68 \%$ by subject AL10I.

TABle I. The Classification Rates Obtained with KNn For Pli

\begin{tabular}{|l|l|l|l|l|l|}
\hline \multirow{2}{*}{ Subjects } & \multicolumn{5}{|l}{ Number of neighbors } \\
\cline { 2 - 6 } & 1 & 2 & 3 & 4 & 5 \\
\hline AB28I & $79,37 \%$ & $79,49 \%$ & $79,59 \%$ & $79,71 \%$ & $79,79 \%$ \\
\hline AS24I & $75,93 \%$ & $76,12 \%$ & $76,15 \%$ & $76,25 \%$ & $76,24 \%$ \\
\hline MA12I & $75,46 \%$ & $75,54 \%$ & $75,71 \%$ & $75,88 \%$ & $75,95 \%$ \\
\hline RR28I & $84,67 \%$ & $84,70 \%$ & $84,69 \%$ & $84,72 \%$ & $84,63 \%$ \\
\hline AL21I & $69,70 \%$ & $69,76 \%$ & $69,88 \%$ & $69,96 \%$ & $69,99 \%$ \\
\hline LA12I & $75,56 \%$ & $75,52 \%$ & $75,38 \%$ & $75,34 \%$ & $75,24 \%$ \\
\hline RH27I & $83,62 \%$ & $83,74 \%$ & $83,83 \%$ & $83,98 \%$ & $84,04 \%$ \\
\hline SA31I & $70,94 \%$ & $70,98 \%$ & $70,95 \%$ & $70,97 \%$ & $70,99 \%$ \\
\hline PD24I & $73,74 \%$ & $73,73 \%$ & $73,70 \%$ & $73,65 \%$ & $73,58 \%$ \\
\hline RA14I & $72,89 \%$ & $72,88 \%$ & $73,03 \%$ & $73,13 \%$ & $73,28 \%$ \\
\hline AL42I & $73,72 \%$ & $73,75 \%$ & $73,74 \%$ & $73,71 \%$ & $73,60 \%$ \\
\hline NA30I & $75,07 \%$ & $75,14 \%$ & $75,18 \%$ & $75,23 \%$ & $75,26 \%$ \\
\hline RS30I & $73,50 \%$ & $73,47 \%$ & $73,43 \%$ & $73,38 \%$ & $73,28 \%$ \\
\hline RV50I & $73,89 \%$ & $73,95 \%$ & $74,07 \%$ & $74,16 \%$ & $74,29 \%$ \\
\hline CA10I & $75,50 \%$ & $75,50 \%$ & $75,50 \%$ & $75,53 \%$ & $75,57 \%$ \\
\hline MC24I & $73,57 \%$ & $73,69 \%$ & $73,78 \%$ & $73,90 \%$ & $73,97 \%$ \\
\hline RA60I & $78,04 \%$ & $78,06 \%$ & $78,13 \%$ & $78,21 \%$ & $78,18 \%$ \\
\hline RG24I & $75,26 \%$ & $75,29 \%$ & $75,25 \%$ & $75,20 \%$ & $75,08 \%$ \\
\hline AL27I & $81,34 \%$ & $81,33 \%$ & $81,31 \%$ & $81,38 \%$ & $81,41 \%$ \\
\hline AP24I & $76,85 \%$ & $76,76 \%$ & $76,76 \%$ & $76,72 \%$ & $76,58 \%$ \\
\hline AC23I & $83,54 \%$ & $83,61 \%$ & $83,68 \%$ & $83,77 \%$ & $83,84 \%$ \\
\hline AL10I & $67,90 \%$ & $67,84 \%$ & $67,76 \%$ & $67,67 \%$ & $67,70 \%$ \\
\hline AL17I & $73,76 \%$ & $73,75 \%$ & $73,70 \%$ & $73,67 \%$ & $73,56 \%$ \\
\hline AL20I & $74,07 \%$ & $74,20 \%$ & $74,27 \%$ & $74,37 \%$ & $74,41 \%$ \\
\hline AL31I & $75,37 \%$ & $75,50 \%$ & $75,59 \%$ & $75,65 \%$ & $75,67 \%$ \\
\hline TR17I & $76,98 \%$ & $76,85 \%$ & $76,80 \%$ & $76,74 \%$ & $76,62 \%$ \\
\hline IB17I & $77,54 \%$ & $77,44 \%$ & $77,39 \%$ & $77,34 \%$ & $77,27 \%$ \\
\hline MIG13I & $80,43 \%$ & $80,45 \%$ & $80,41 \%$ & $80,33 \%$ & $80,15 \%$ \\
\hline RC13I & $75,28 \%$ & $75,33 \%$ & $75,52 \%$ & $75,63 \%$ & $75,79 \%$ \\
\hline CM28I & $73,46 \%$ & $73,52 \%$ & $73,43 \%$ & $73,44 \%$ & $73,36 \%$ \\
\hline MF41I & $71,74 \%$ & $71,77 \%$ & $71,94 \%$ & $72,04 \%$ & $72,24 \%$ \\
\hline RE60I & $76,43 \%$ & $76,46 \%$ & $76,41 \%$ & $76,35 \%$ & $76,28 \%$ \\
\hline & & & & & \\
\hline
\end{tabular}

Using WPLI, the classification rates for all the subjects are smaller with $20 \%$ in comparison with PLI using classifiers LDA, QDA, MD (Fig.2). The accuracy rates are below $66 \%$.

Unlike PLI, where none of the subjects obtained higher classification rates with LDA classifier, for WPLI, 6 subjects obtained the best classification rates with LDA, 18 subjects with QDA classifier and 9 subjects with MD classifier.

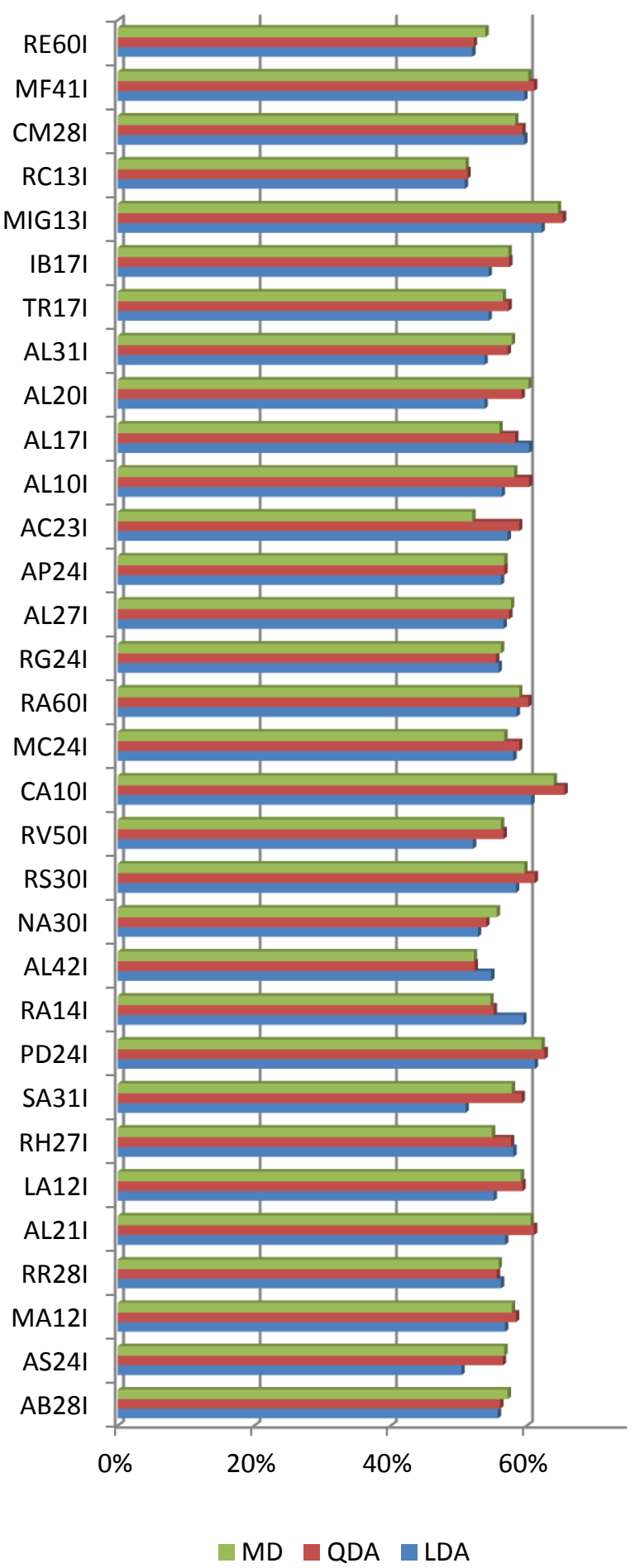

Fig. 2. The classification rates (\%) for WPLI using classifiers LDA, QDA and MD 
Subjects MIG13I and CA10I achieved the highest classification rates by means of KNN classifier when WPLI is used as feature vector. In comparison with PLI the classification rates obtained with WPLI are also lower (TABLE II).

TABLE II. THE Classification RATES OBTAINED WITH KNN FOR WPLI

\begin{tabular}{|l|l|l|l|l|l|}
\hline \multirow{2}{*}{ Subjects } & Number of neighbors & \multicolumn{5}{l|}{} \\
\cline { 2 - 6 } & 1 & 2 & 3 & 4 & 5 \\
\hline AB28I & $53,72 \%$ & $53,79 \%$ & $54,05 \%$ & $54,22 \%$ & $54,30 \%$ \\
\hline AS24I & $53,56 \%$ & $53,33 \%$ & $53,31 \%$ & $53,18 \%$ & $53,12 \%$ \\
\hline MA12I & $56,36 \%$ & $56,51 \%$ & $56,67 \%$ & $56,80 \%$ & $56,77 \%$ \\
\hline RR28I & $53,30 \%$ & $53,27 \%$ & $53,31 \%$ & $53,38 \%$ & $53,43 \%$ \\
\hline AL21I & $54,95 \%$ & $54,95 \%$ & $54,93 \%$ & $54,93 \%$ & $54,85 \%$ \\
\hline LA12I & $57,58 \%$ & $57,55 \%$ & $57,53 \%$ & $57,53 \%$ & $57,50 \%$ \\
\hline RH27I & $54,96 \%$ & $54,96 \%$ & $54,95 \%$ & $54,98 \%$ & $54,99 \%$ \\
\hline SA31I & $58,36 \%$ & $58,51 \%$ & $58,54 \%$ & $58,60 \%$ & $58,58 \%$ \\
\hline PD24I & $59,27 \%$ & $59,26 \%$ & $59,25 \%$ & $59,28 \%$ & $59,27 \%$ \\
\hline RA14I & $54,34 \%$ & $54,42 \%$ & $54,51 \%$ & $54,66 \%$ & $54,66 \%$ \\
\hline AL42I & $51,89 \%$ & $51,71 \%$ & $51,61 \%$ & $51,46 \%$ & $51,34 \%$ \\
\hline NA30I & $55,15 \%$ & $55,34 \%$ & $55,45 \%$ & $55,55 \%$ & $55,70 \%$ \\
\hline RS30I & $57,73 \%$ & $57,75 \%$ & $57,70 \%$ & $57,75 \%$ & $57,83 \%$ \\
\hline RV50I & $55,13 \%$ & $55,08 \%$ & $55,05 \%$ & $55,01 \%$ & $55,01 \%$ \\
\hline CA10I & $61,58 \%$ & $61,63 \%$ & $61,60 \%$ & $61,63 \%$ & $61,56 \%$ \\
\hline MC24I & $57,38 \%$ & $57,43 \%$ & $57,28 \%$ & $57,26 \%$ & $57,18 \%$ \\
\hline RA60I & $56,21 \%$ & $55,95 \%$ & $55,83 \%$ & $55,69 \%$ & $55,49 \%$ \\
\hline RG24I & $52,39 \%$ & $52,43 \%$ & $52,41 \%$ & $52,39 \%$ & $52,37 \%$ \\
\hline AL27I & $57,35 \%$ & $57,46 \%$ & $57,68 \%$ & $57,97 \%$ & $58,21 \%$ \\
\hline AP24I & $55,07 \%$ & $55,18 \%$ & $55,37 \%$ & $55,54 \%$ & $55,62 \%$ \\
\hline AC23I & $54,47 \%$ & $54,50 \%$ & $54,55 \%$ & $54,60 \%$ & $54,70 \%$ \\
\hline AL10I & $56,15 \%$ & $56,12 \%$ & $56,02 \%$ & $56,00 \%$ & $55,92 \%$ \\
\hline AL17I & $57,52 \%$ & $57,47 \%$ & $57,42 \%$ & $57,34 \%$ & $57,22 \%$ \\
\hline AL20I & $57,84 \%$ & $57,87 \%$ & $57,95 \%$ & $58,02 \%$ & $57,99 \%$ \\
\hline AL31I & $55,21 \%$ & $55,34 \%$ & $55,41 \%$ & $55,48 \%$ & $55,45 \%$ \\
\hline TR17I & $52,11 \%$ & $52,16 \%$ & $52,08 \%$ & $52,00 \%$ & $51,91 \%$ \\
\hline IB17I & $54,52 \%$ & $54,57 \%$ & $54,53 \%$ & $54,49 \%$ & $54,44 \%$ \\
\hline MIG13I & $62,81 \%$ & $62,70 \%$ & $62,71 \%$ & $62,70 \%$ & $62,75 \%$ \\
\hline RC13I & $54,13 \%$ & $54,14 \%$ & $54,11 \%$ & $54,10 \%$ & $54,03 \%$ \\
\hline CM28I & $57,80 \%$ & $57,79 \%$ & $57,76 \%$ & $57,77 \%$ & $57,73 \%$ \\
\hline MF41I & $58,60 \%$ & $58,56 \%$ & $58,52 \%$ & $58,37 \%$ & $58,23 \%$ \\
\hline RE60I & $54,74 \%$ & $54,60 \%$ & $54,45 \%$ & $54,28 \%$ & $54,08 \%$ \\
\hline & & & & & \\
\hline
\end{tabular}

Fig. 3 presents the results for PLI compared with the results for WPLI using SVM classifier. For PLI, the highest classification rate is $86.25 \%$ - subject RR28I and for WPLI $61.56 \%$ - subject AL27I.

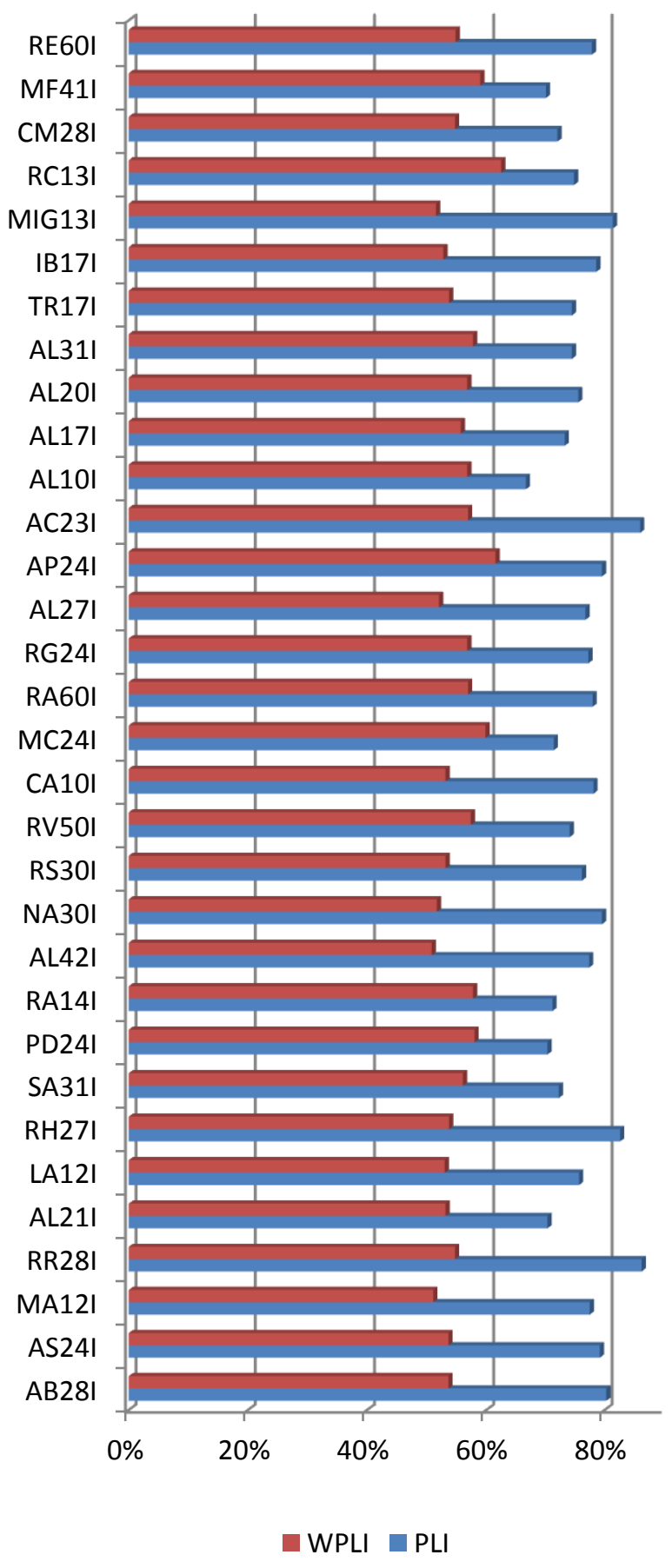

Fig. 3. The classification rates for PLI and WPLI using SVM classifier 
In the next section are shown the classification results obtained on the second dataset used. Subject 1 achieved the following discrimination rates: $85.80 \%$ - LDA, 83.33\% - QDA and $87.08 \%$ - MD for PLI (Fig. 4).

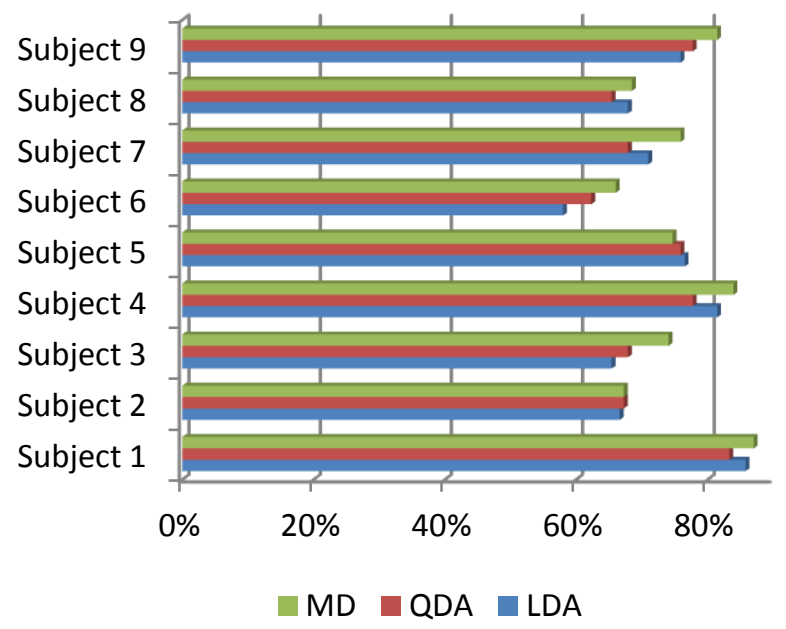

Fig. 4. The classification rates for PLI applying LDA, QDA and MD

KNN classification with 1, 2, 3, 4, 5 neighbors is depicted in TABLE III. The results are in the range of $63.99 \%$ (subject 8) and $87.34 \%$ (subject 1 ).

TABLE III. THE ClASSIFICATION RATES (\%) OBTAINED WITH KNN FOR PLI

\begin{tabular}{|l|l|l|l|l|l|}
\hline \multirow{2}{*}{ Subjects } & \multicolumn{5}{|l}{ Number of neighbors } \\
\cline { 2 - 6 } & 1 & 2 & 3 & 4 & 5 \\
\hline Subject 1 & $87,22 \%$ & $87,34 \%$ & $87,34 \%$ & $87,34 \%$ & $87,24 \%$ \\
\hline Subject 2 & $68,94 \%$ & $68,72 \%$ & $68,51 \%$ & $68,31 \%$ & $68,11 \%$ \\
\hline Subject 3 & $72,36 \%$ & $72,29 \%$ & $72,13 \%$ & $71,97 \%$ & $71,91 \%$ \\
\hline Subject 4 & $85,68 \%$ & $85,82 \%$ & $85,85 \%$ & $85,98 \%$ & $85,91 \%$ \\
\hline Subject 5 & $77,75 \%$ & $77,49 \%$ & $77,34 \%$ & $77,20 \%$ & $77,26 \%$ \\
\hline Subject 6 & $70,26 \%$ & $70,02 \%$ & $69,68 \%$ & $69,46 \%$ & $69,24 \%$ \\
\hline Subject 7 & $72,47 \%$ & $72,40 \%$ & $72,23 \%$ & $72,18 \%$ & $72,02 \%$ \\
\hline Subject 8 & $65,20 \%$ & $65,04 \%$ & $64,79 \%$ & $64,44 \%$ & $63,99 \%$ \\
\hline Subject 9 & $84,47 \%$ & $84,31 \%$ & $84,36 \%$ & $84,31 \%$ & $84,26 \%$ \\
\hline
\end{tabular}

The discrimination rates achieved with WPLI and LDA, QDA and MD are presented in Fig. 5. For each of the three classifiers, the results are smaller compared to those when the feature vector used the PLIs.

In TABLE IV. are presented the results obtained with KNN. The higher classification is achieved by Subject 9 and the smaller one by Subject 3.

Concerning the comparison between performances achieved with SVM classifier, for PLI and WPLI the higher difference between rates is $29.01 \%$ for subject 1 and the smaller one is $1.85 \%$ (Fig. 6). Subject 7 attained a better classification for WPLI.

The findings are consistent with other works [18], [19] in which the second dataset is exploited. So, in [18] where a timefrequency approach is investigated using six subjects $(1,2,5$, $6,7,9$ ), for subject 1 and subject 9 are reported the classification rates $81.11 \%$ and $83.61 \%$, respectively. With PLI for subject 1 the classification rates achieved are in the range $77.78 \%-87.34 \%$, while for subject 9 the classification rates were in the range $67.28 \%-84.47 \%$. For WPLI, for subject 1 the classification rates are between $48.76 \%-62.34 \%$ and for subject 9: $59.25 \%-62.86 \%$.

In [20], where Itakura distance based method is used and 20 subjects from the first database, the classification rates are approximately the same with the actual results. For example, subject RR28I achieved 81.67\%, 86.67\%, 85.00\% classification rates using LDA, QDA and MD classifiers, compared to $74.09 \%, 79.68 \%, 79.56 \%$ for PLI and $56.20 \%$, $55.60 \%$ and $55.84 \%$ for WPLI.

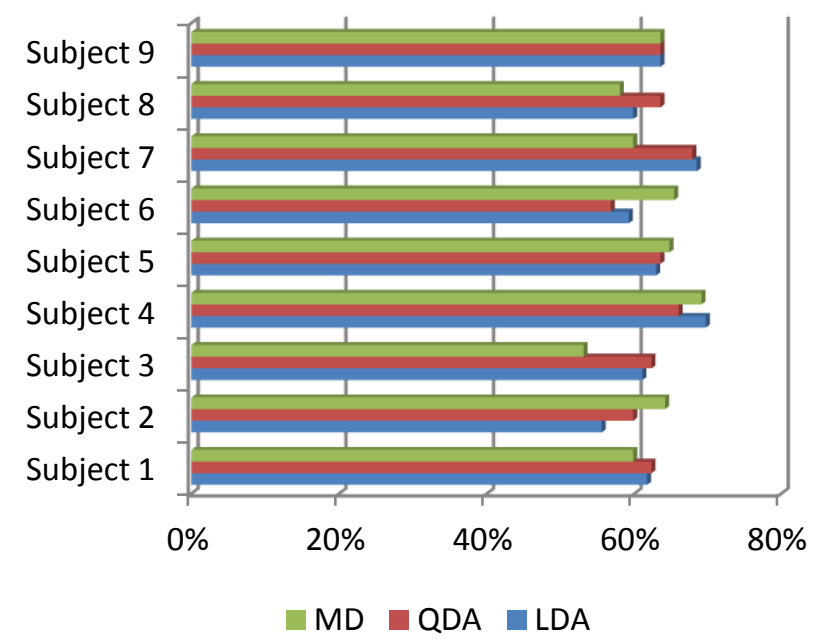

Fig. 5. LDA, QDA and MD classification corresponding to the WPLI method

TABLE IV. The Classification RATES OBtAINED WITH KNN FOR WPLI

\begin{tabular}{|l|l|l|l|l|l|}
\hline \multirow{2}{*}{ Subjects } & \multicolumn{5}{|l}{ Number of neighbors } \\
\cline { 2 - 6 } & 1 & 2 & 3 & 4 & 5 \\
\hline Subject 1 & $61,78 \%$ & $61,69 \%$ & $61,70 \%$ & $61,51 \%$ & $61,63 \%$ \\
\hline Subject 2 & $56,50 \%$ & $56,39 \%$ & $56,49 \%$ & $56,49 \%$ & $56,17 \%$ \\
\hline Subject 3 & $55,18 \%$ & $55,19 \%$ & $55,11 \%$ & $55,13 \%$ & $55,35 \%$ \\
\hline Subject 4 & $62,00 \%$ & $61,90 \%$ & $61,70 \%$ & $61,30 \%$ & $61,32 \%$ \\
\hline Subject 5 & $62,56 \%$ & $62,45 \%$ & $62,45 \%$ & $62,45 \%$ & $62,55 \%$ \\
\hline Subject 6 & $59,47 \%$ & $59,63 \%$ & $59,68 \%$ & $59,62 \%$ & $59,67 \%$ \\
\hline Subject 7 & $59,03 \%$ & $58,77 \%$ & $58,72 \%$ & $58,68 \%$ & $58,54 \%$ \\
\hline Subject 8 & $57,05 \%$ & $57,03 \%$ & $57,02 \%$ & $57,01 \%$ & $57,00 \%$ \\
\hline Subject 9 & $62,11 \%$ & $62,34 \%$ & $62,55 \%$ & $62,87 \%$ & $62,86 \%$ \\
\hline
\end{tabular}




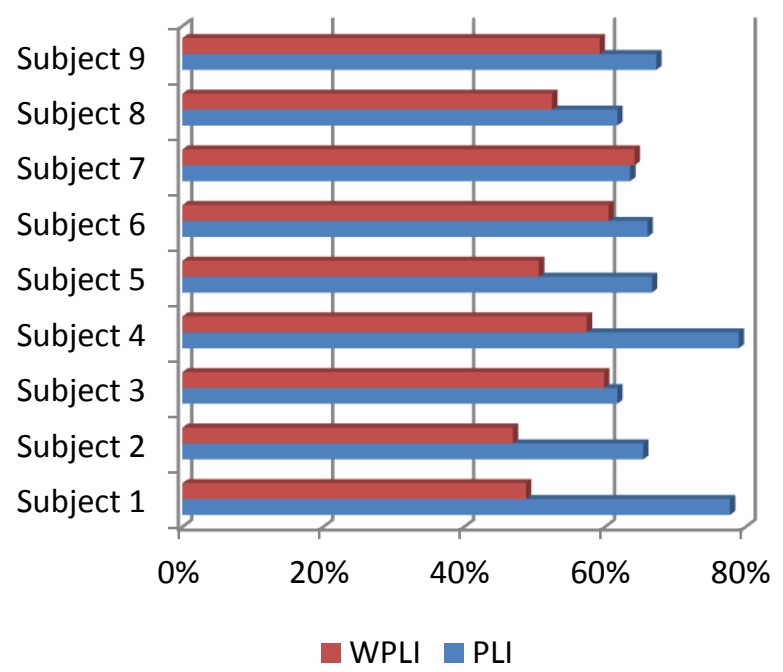

Fig. 6. The classification rates for PLI and WPLI using SVM classifier

Fig. 7 displays the highest classification rates obtained for phase lag index and weighted phase lag index with all five classifiers and for datasets used.

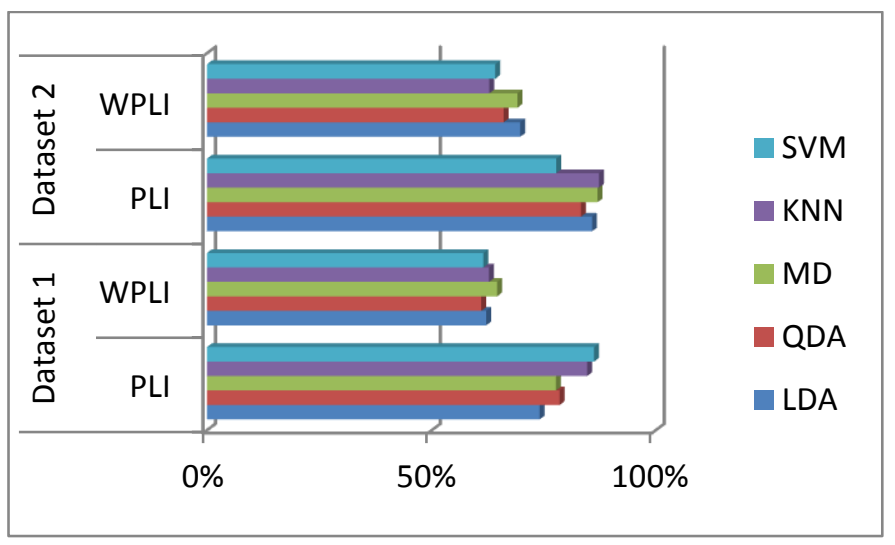

Fig. 7. The classification accuracies using all classifiers for PLI and WPLI

\section{DISCUSSIONS}

The research evaluated two phase synchronization based methods with two indexes: phase lag index and weighted phase lag index, on two different datasets. Phase synchronization contains important features connected to the brain activity and in this way the discrimination can be made between left and right hand movement. The first dataset is formed by EEG recordings collected by the authors. So, in this case, the research is more efficient because all the details and conditions on which the recordings were made are known. The opinion of the voluntaries and their information regarding the experiments are also well-known. The volunteers are trained first by performing effectively the movement of the left/right hand and after that performing left/right hand imagination. For the second dataset used there are not available so many information: the acquisition system it is not described or mentioned, isn't specified if the recordings took placed in the same day or in different days and the age of the volunteers is not known. It is mentioned that the subjects were well trained. The technical and social details are very important in developing a brain computer interface.

For the first dataset, the classification rates obtained with PLI are different from the results attained with WPLI. Using LDA classification method 5 subjects obtained differences higher with $10 \%$ for PLI in comparison with WPLI. Two subjects achieved better results with WPLI. Differences below 5\% between PLI and WPLI were noticed at 8 subjects. With QDA classifier classification rates obtained are higher than LDA but also the higher differences between classification rates for PLI and WPLI. For 70\% of the subjects the differences between classification rates are in the range of $10.22 \%-19.71 \%$. No subject has achieved higher classification rates for WPLI. MD classification provided better results for PLI for $87 \%$ of the subjects. The smaller difference between the classification rates is obtained by one subject $-2.07 \%$.

If we are comparing the results obtained with LDA, QDA and MD the higher classifications rates are achieved with QDA.

Regarding the KNN classification the number of neighbors is very important. We have chosen to represent all five neighbors in order to see which neighbor is the best. The average for 1 neighbor was $75.78 \%, 2$ neighbors $-75.82,3$ neighbors $-75.84 \%, 4$ neighbors $-75.88 \%$, 5 neighbors $75.87 \%$ in the case of PLI. In the case of WPLI the average obtained were $56.01 \%, 56.01 \%, 56.02 \%, 56.03 \%, 56.01 \%$. SVM classifier outperforms all classifiers used. In this cases aren't highlighted major differences between neighbors. Two subjects achieved classification rates above $86 \%$ when the feature vector is formed with and other two subjects when the feature vector is formed with WPLI.

For the second dataset used we can conclude the following: the higher difference between PLI and WPLI is $24.07 \%$ and the smaller difference is $2.47 \%$ for LDA classification.

QDA method revealed approximately the same results as LDA classification. The highest classification rate is about $83.33 \%$ and the smallest one is about $62.35 \%$.

If we compare LDA, QDA and MD the higher classification rates are obtained with MD. For PLI the results are also better.

In KNN classification the difference between neighbors were not significant. The average was $76 \%$ for PLI and $56 \%$ for WPLI.

With SVM classification the highest classification rates are also obtained.

Overall, the classification rates are better for the second dataset.

The discrimination rates are higher with $8-10 \%$ for the second dataset compared to the first dataset.

Although WPLI is a more complex method by introducing weighted normalized phase difference, the classification rate is not higher than $60 \%$. 
The type of classifier utilized, the way how a subject could imagine the hand movement are some aspects that should be explored more deeply.

\section{CONCLUSIONS AND FUTURE WORK}

Offline analysis based on phase synchronization are proposed and tested.

The results suggest that methods could be exploited for BCI paradigms. It was found a large variability between subjects and between datasets.

PLI and WPLI avoid artifacts caused by the volume conductor. Volume conductor is very important for the EEG recordings because the analysis of connectivity is limited due to the low spatial resolution.

The results revealed satisfactory even although no methods for elimination of artifacts and few EEG channels were used.

Future wok implies developing an ensemble classifier (a combination of classification methods) for improving the classification rates.

\section{ACKNOWLEDGMENT}

This work was supported by a grant of the Romanian National Authority for Scientific Research and Innovation, CNCS - UEFISCDI, project number PN-II-RU-TE-2014-40832 "Medical signal processing methods based on compressed sensing; applications and their implementation".

\section{REFERENCES}

[1] J.R. Wolpaw, N. Birbaumer, D.J. McFarland, G. Pfurtscheller, T.M. Vaughan, "Brain-computer interfaces for communication and control", Clinical Neuro- physiology, vol. 113(6), pp. 767-791, 2002.

[2] Y.Wang, S. Gao, X. Gao, "Common spatial pattern method for channel selelction in motor imagery based brain-computer interface", IEEE Engineering in Medicine and Biology 27th Annual Conference, pp. 5392-5395, 2005.

[3] A. Schlögl et al., "Characterization of four-class motor imagery EEG data for the BCI-competition 2005", Journal of neural engineering, , 2.4: L14, 2005.

[4] R. Aldea, D. Tarniceriu, "Estimating the Hurst exponent in motor imagery-based brain computer interface", Speech Technology and Human-Computer Dialogue (SpeD), IEEE, pp. 1-6, 2013.

[5] I. Daly, S.J. Nasuto, K. Warwick, "Brain computer interface control via functional connectivity dynamics", Pattern recognition, vol. 45(6), pp. 2123-2136, 2012
[6] F. Lotte, M. Congedo, A. Le'cuyer, F. Lamarche, B. Arnaldi, "A review of classification algorithms for EEG-based brain-computer interfaces", Journal of NeuralEngineering, 4(2), 2007.

[7] M. Vinck, R. Oostenveld et al., "An improved index of phasesynchronization for electrophysiological data in the presence of volumeconduction, noise and sample-size bias", Neuroimage, vol. 55(4), pp. 1548-1565, 2011.

[8] JP Lachaux, E. Rodriguez et al., "Measuring phase synchrony in brain signals”, Hum Brain Mapp., vol. 8(4), pp. 194-208, 1999.

[9] G. Nolte, O. Bai, L. Wheaton, Z. Mari, S. Vorbach, M. Hallett, "Identifying true brain interaction from EEG data using the imaginary part of coherency", Clin. Neurophysiol., vol. 115, pp. 2292-2307, 2004.

[10] C.J. Stam, G. Nolte, A. Daffertshofer, "Phase lag index: assessment of functional connectivity from multi channel EEG and MEG with diminished bias from common sources", Hum. Brain Mapp., vol. 28, pp. 1178-1193, 2007.

[11] C. Neuper, R. Scherer, S. Wriessneger, G. Pfurtscheller, "Motor imagery and action observation: modulation of sensorimotor brain rhythms during mental control of a brain-computer interface", Clinical Neurophysiology, vol. 120, pp. 239-247, 2009.

[12] J. Mellinger, G. Schalk: BCI2000: A General-Purpose Software Platform for BCI Research, In: G. Dornhege, J. del R. Millán, T. Hinterberger, D.J. McFarland, K.-R. Müller (eds.), Toward BrainComputer Interfacing, MIT Press, 2007.

[13] A. Osman, A. Robert., "Time-course of cortical activation during overt and imagined movements", Proc. Cognitive Neuroscience Annu. Meet., New York vol. 1, pp. 1842-1852, 2001.

[14] T. Hastie, R. Tibshirani, J. Friedman, "The Elements of Statistical Learning - Data Mining, Inference and Prediction", Springer Series in Statistics, pp.110-111, 2009.

[15] F. Babiloni, L. Bianchi, F. Semeraro, J. del R Millan, J. Mouriño, A. Cattini, F. Cincotti, "Mahalanobis distance-based classifiers are able to recognize EEG patterns by using few EEG electrodes", Engineering in Medicine and Biology Society, 1, pp. 651-654,2001.

[16] W.A. Chaovalitwongse, Y.J. Fan, R.C. Sachdeo, "On the time series knearest neighbor classification of abnormal brain activity", Systems, Man and Cybernetics, Part A: Systems and Humans, IEEE Transactions, vol. 6, pp. 1005-1016, 2007

[17] K.P. Bennett, C. Campbell, "Support vector machines: hype or hallelujah?", ACM SIGKDD Explorations Newsletter, vol. 2(2), pp.1$13,2000$.

[18] N. F. Ince, A.H. Tewfik, S. Arica, "Extraction subject-specific motor imagery time-frequency patterns for single trial EEG classification", Computers in Biology and Medicine, vol. 37(4), pp. 499-508, 2007.

[19] B. Kamousi, Z. Liu, B. He, "An EEG inverse solution based braincomputer interface", The International Journal of Bioelectromagnetism, vol. 7(2), pp. 292-294, 2005.

[20] O.D. Eva, A.M. Lazar, "Channels selection for motor imagery paradigm - An Itakura distance based method", E-Health and Bioengineering Conference (EHB), IEEE, pp. 1-4, 2015. 